\title{
Effect of Polyethylene Glycol Induced Water Stress on Germination and Seedling Growth of Wheat (Triticum aestivum)
}

\author{
M.S. Rana, M.A. Hasan*, M.M. Bahadur and M.R. Islam \\ Department of Crop Physiology and Ecology \\ Hajee Mohammad Danesh Science and Technology University, Dinajpur-5200, Bangladesh. \\ *Corresponding author and Email: mdabuhasan@yahoo.com
}

Received: 20 January 2017

Accepted: 12 June 2017

\begin{abstract}
The performance of twenty wheat genotypes under Polyethylene Glycol (PEG) induced water stress during germination and early seedling growth stages were tested under three levels of water potential i) Control (Tap water), ii) -2 bars and iii) -4 bar at the Crop Physiology and Ecology Laboratory of Hajee Mohammad Danesh Science and Technology University, Dinajpur during September 2014 to October 2014. Rate of germination and vigor index of all wheat genotypes were delayed with the increment of water stress induced by PEG. Shoot and root lengths and seedling dry weight of 10 days old seedlings were found to be reduced due to the increment of water stress. However, the degree of reduction of these parameters with the increment of water stress was not similar for all wheat genotypes. Stress tolerance index (STI) based on seedling dry weight indicated a wide difference in stress tolerance among the wheat genotypes. At moderate water deficit stress, BARI Gom 25, E 34, E 28 and BAW 1170 showed more stress tolerance and the wheat genotypes- Sourav, E 23 and BAW 1140 showed greater stress sensitivity than the other wheat genotypes. At higher water deficit stress, BARI Gom 25, BARI Gom 28, E 28 and BAW 1170 showed more stress tolerance and the wheat genotypes- Satabdi, Sourav, BARI Gom 26, E 23, E 38, E 24, BAW 1163, BAW 1140 and BAW 1151 showed greater stress sensitivity than the others. Considering both moderate and high water deficit stress, BARI Gom 25, E 28 and BAW 1170 were found as tolerant and Sourav, E 23 and BAW 1140 were found as water deficit stress sensitive wheat genotypes.
\end{abstract}

Key words: PEG, Stress tolerance index, germination characteristics and wheat.

\section{Introduction}

Wheat (Triticum aestivum), next to rice is the staple food of the peoples of Bangladesh grown over an area of 0.43 million hectare with an annual production of about 1.303 million metric tons at average of $3.03 \mathrm{t} \mathrm{ha}^{-1}$ (Anonymous, 2014). Its consumption has been increasing with changing food habit in our country. The average production of this crop in Bangladesh is low compared to other wheat growing countries because wheat is mainly grown under nonirrigated conditions during the dry winter (November to April) in Bangladesh (Hossain and Silva, 2013). Although the vast storage of soil moisture resulted from monsoon rain supports the plant growth favorably at the early stages of growth, the plant suffers from water stress at the reproductive stage when the residual soil moisture depletes (Lopez et al., 2003). 
Polyethylene glycol widely used to induce water stress in plants is a non-ionic water soluble polymer which is not expected to penetrate into cells (Djibril et al., 2005). Selection for drought tolerance at early stage of seedlings is most frequently practiced using poly ethylene glycol (PEG 6000) in the medium (Rauf et al., 2006). Earlier reports on identification of the drought tolerant wheat genotypes using different concentrations of PEG 6000 have showed significant differences for different seedling traits (Rauf et al., 2006, Singh et al., 2008). The seedling traits when pooled together could discriminate between drought tolerant and susceptible genotypes (Noorka and Khaliq, 2007). Therefore, to expand wheat cultivation and to sustain wheat yield under drought prone areas the present investigation was carried out to evaluate germination characters and seedling growth of wheat as screening criteria against drought stress.

\section{Materials and Methods}

\subsection{Location and duration}

The experiment was conducted at Crop Physiology and Ecology Laboratory, Hajee Mohammad Danesh Science and Technology University, Dinajpur during September 2014 to October 2014.

\subsection{Experimental design and treatments}

The experiment was carried out in completely randomized design (factorial) with three replications. One factor consisted of three levels of water potential- Control (Tap water), moderate stress (-2 bars induced PEG 6000) and higher stress ( -4 bars induced by PEG 6000) and the other factor consisted of twenty wheat genotypes including advance lines and popular varieties (Table 1).

\subsection{Collection and placement of seed}

Seeds of twenty wheat genotypes (Table 1) collected from Wheat Research Centre of BARI, Dinajpur, Bangladesh were surface sterilized by dipping the seeds in $0.1 \%$ mercuric chloride solution for 2 minutes and rinsed thoroughly with sterilized water. Required amount of PEG 6000 was dissolved in tap water as described by (Michel, 1983) to develop -2 bars and -4 bar water potential. Thirty seeds of each genotype were placed for germination in $11 \mathrm{cmpetridish}$ on sand bed irrigated with respective treatments. The petridishes were irrigated daily with required amount $(5 \mathrm{ml})$ of respective solution. Each treatment was replicated thrice. Seedlings were allowed to grow up to 7 days after placement for germination.

\subsection{Data recorded and analysis}

Germination was counted at 24-hour interval and continued upto $7^{\text {th }}$ day (168 hrs). A seed was considered germinated as plumule and radicle came out and were $>2 \mathrm{~mm}$ long. The rate of germination was calculated using the formula as described by Krishnasamy and Seshu (1990) and co-efficient of germination and vigor index were calculated using the formulae as described by Copeland (1976). At 7th day after placement for germination, all other seedlings were removed from each petridish keeping 10 healthy seedlings and allowed to grow upto 10 days by adding required amount $(5 \mathrm{ml})$ of respective solution daily. At 10 days after placement for germination, five seedlings from each petridish were sampled. Shoot and root length of individual seedling were recorded manually with scale. Then the seedlings were dried separately at $70^{\circ} \mathrm{C}$ for $72 \mathrm{~h}$ in an electric oven (Model- E28\# 03-54639, Binder, Germany) and weight were recorded with an electrical balance (Model- EK$300 \mathrm{i})$. The mean length $(\mathrm{cm})$ and dry weight were recorded for each treatment combination. Stress tolerance index was calculated as Goudarzi and Pakniyat (2008). The data were analyzed by partitioning the total variance with the help of computer using MSTAT program.

\section{Results and Discussion}

\subsection{Germination character \\ 3.1.1 Rate of germination}

Rate of germination which indicates the speed of germination was significantly influenced by the interaction effect of water potential levels and 
wheat genotypes during germination (Table 2). Rate of germination was higher at control (with a range from 82.66 in BAW 1170 to 98.66 in Satabdi and BARI Gom 27 and a mean of 93.28), moderate at moderate stress (with a range from 77.00 in BAW 1140 to 95.66 in E 34 and a mean of 88.36) and lower at higher water deficit stress (with a range from 63.33 in BAW 1140 to 91.33 in E 34 and a mean of 81.44). The results of Table 2 showed that the speed of germination was reduced with the increment of water deficit stress but the degree of reduction in rate of germination was not similar for all wheat genotypes at moderate and higher water deficit stress compared to control. At moderate water deficit stress, wheat genotypes- BARI Gom 25, BARI Gom 26, BARI Gom 28, E 30, E 2, E 24, E 34, BAW 1138, BAW 1170 and BAW 1151 showed less than $5 \%$ reduction and wheat genotypes- Satabdi, Sourav, BARI Gom 27, E 23, E 38, E 28, BAW 1171, BAW 1157, BAW 1163 and BAW 1140 showed more than 5\% reduction in rate of germination compared to control. At higher water deficit stress, wheat genotypes- Sourav, BARI Gom 25, BARI Gom 26, E 30, E 2 and E 34 showed less 10\% reduction and wheat genotypes- Satabdi, BARI Gom 27, BARI Gom 28, E 23, E 38, E 24, E 28, BAW 1138, BAW 1171, BAW 1170, BAW 1157, BAW 1163, BAW 1140 and BAW 1151 showed more than $10 \%$ reduction in rate of germination compared to control.

\subsubsection{Germination vigor index}

Different levels of water potential interacted significantly to wheat genotypes in influencing vigor index which also expresses the speed of germination (Table 2).

Table 1. List of wheat genotypes used for the present study

\begin{tabular}{lll}
\hline Sl. No. & Genotypes & Remarks \\
\hline 1. & Shatabdi & Variety \\
2. & Sourab & Variety \\
3. & BARI Gom 25 & Variety \\
4. & BARI Gom 26 & Variety \\
5. & BARI Gom 27 & Variety \\
6. & BARI Gom 28 & Variety \\
7. & E 30 & Advanced line \\
8. & E 23 & Advanced line \\
9. & E 2 & Advanced line \\
10. & E 38 & Advanced line \\
11. & E 24 & Advanced line \\
12. & E 34 & Advanced line \\
13. & E 28 & Advanced line \\
14. & BAW1138 & Advanced line \\
15. & BAW1170 & Advanced line \\
16. & BAW1157 & Advanced line \\
17. & BAW1157 & Advanced line \\
18. & BAW1163 & Advanced line \\
19. & BAW1140 & Advanced line \\
20. & BAW1151 & Advanced line \\
\hline
\end{tabular}


Table 2. Rate of germination and vigor index of different wheat genotypes as influenced PEG induced water deficit stress

\begin{tabular}{|c|c|c|c|c|c|c|}
\hline \multirow[b]{2}{*}{ Genotypes } & \multicolumn{3}{|c|}{ Rate of germination (mean $\pm \mathrm{SE}$ ) } & \multicolumn{3}{|c|}{ Vigor index $($ mean $\pm \mathrm{SE})$} \\
\hline & $\begin{array}{c}\text { Control } \\
\text { (Tap water) }\end{array}$ & $\begin{array}{l}\text { Moderate } \\
\text { stress } \\
(-2 \text { bar })\end{array}$ & $\begin{array}{l}\text { Higher stress } \\
\text { (- 4bar) }\end{array}$ & $\begin{array}{c}\text { Control } \\
\text { (Tap water) }\end{array}$ & $\begin{array}{c}\text { Moderate } \\
\text { stress } \\
(-2 \text { bar })\end{array}$ & $\begin{array}{l}\text { Higher stress } \\
\text { (- 4bar) }\end{array}$ \\
\hline Satabdi & $98.66 \pm 1.70$ & $\begin{array}{c}93.00 \pm 1.09 \\
(-5.74)\end{array}$ & $\begin{array}{c}87.33 \pm 2.18 \\
(-11.48)\end{array}$ & $43.32 \pm 1.83$ & $\begin{array}{c}37.49 \pm 1.28 \\
(-13.46)\end{array}$ & $\begin{array}{c}36.88 \pm 0.87 \\
(-14.87)\end{array}$ \\
\hline Sourav & $90.00 \pm 1.01$ & $\begin{array}{c}83.33 \pm 1.49 \\
(-7.41)\end{array}$ & $\begin{array}{c}82.66 \pm 1.47 \\
(-8.16)\end{array}$ & $36.47 \pm 0.48$ & $\begin{array}{c}34.17 \pm 2.17 \\
\quad(-6.31)\end{array}$ & $\begin{array}{c}33.97 \pm 0.48 \\
\quad(-6.86)\end{array}$ \\
\hline $\begin{array}{l}\text { BARI Gom } \\
25\end{array}$ & $97.33 \pm 3.05$ & $\begin{array}{c}94.66 \pm 0.62 \\
(-2.74)\end{array}$ & $\begin{array}{c}88.66 \pm 4.98 \\
(-8.91)\end{array}$ & $38.98 \pm 0.91$ & $\begin{array}{c}38.02 \pm 1.00 \\
(-2.46)\end{array}$ & $\begin{array}{c}37.73 \pm 0.91 \\
\quad(-3.21)\end{array}$ \\
\hline $\begin{array}{l}\text { BARI Gom } \\
26\end{array}$ & $97.33 \pm 2.40$ & $\begin{array}{c}94.33 \pm 1.41 \\
(-3.08)\end{array}$ & $\begin{array}{c}88.00 \pm 1.56 \\
(-9.59)\end{array}$ & $41.48 \pm 0.87$ & $\begin{array}{c}37.57 \pm 0.34 \\
(-9.43)\end{array}$ & $\begin{array}{c}37.22 \pm 0.34 \\
(-10.27)\end{array}$ \\
\hline $\begin{array}{l}\text { BARI Gom } \\
27\end{array}$ & $98.66 \pm 2.00$ & $\begin{array}{c}89.33 \pm 2.33 \\
(-9.46)\end{array}$ & $\begin{array}{c}86.66 \pm 4.00 \\
(-12.16)\end{array}$ & $38.94 \pm 1.00$ & $\begin{array}{c}38.47 \pm 1.28 \\
\quad(-1.21)\end{array}$ & $\begin{array}{c}37.96 \pm 0.18 \\
(-2.52)\end{array}$ \\
\hline $\begin{array}{l}\text { BARI Gom } \\
28\end{array}$ & $93.66 \pm 1.33$ & $\begin{array}{c}91.66 \pm 6.67 \\
(-2.14)\end{array}$ & $\begin{array}{c}78.66 \pm 2.60 \\
(-16.02)\end{array}$ & $38.93 \pm 1.25$ & $\begin{array}{c}36.87 \pm 0.91 \\
(-5.29)\end{array}$ & $\begin{array}{c}34.03 \pm 0.34 \\
(-12.59)\end{array}$ \\
\hline E 30 & $94.33 \pm 0.54$ & $\begin{array}{c}93.00 \pm 0.77 \\
(-1.41)\end{array}$ & $\begin{array}{c}89.66 \pm 0.83 \\
(-4.95)\end{array}$ & $42.76 \pm 2.17$ & $\begin{array}{c}40.86 \pm 0.72 \\
(-4.44)\end{array}$ & $\begin{array}{c}36.01 \pm 0.87 \\
(-15.79)\end{array}$ \\
\hline Е 23 & $94.00 \pm 1.14$ & $\begin{array}{c}86.66 \pm 2.60 \\
(-7.81)\end{array}$ & $\begin{array}{c}83.33 \pm 1.20 \\
(-11.35)\end{array}$ & $39.62 \pm 0.34$ & $\begin{array}{c}37.43 \pm 1.25 \\
(-5.53)\end{array}$ & $\begin{array}{c}34.94 \pm 0.76 \\
(-11.81)\end{array}$ \\
\hline E 2 & $91.00 \pm 0.83$ & $\begin{array}{c}89.66 \pm 0.54 \\
(-1.47)\end{array}$ & $\begin{array}{c}85.00 \pm 0.77 \\
(-6.59)\end{array}$ & $41.59 \pm 0.18$ & $\begin{array}{c}40.51 \pm 0.76 \\
(-2.60)\end{array}$ & $\begin{array}{c}36.71 \pm 0.18 \\
(-11.73)\end{array}$ \\
\hline E 38 & $90.66 \pm 0.77$ & $\begin{array}{c}85.28 \pm 0.11 \\
(-5.93)\end{array}$ & $\begin{array}{c}71.88 \pm 0.83 \\
(-20.71)\end{array}$ & $38.01 \pm 0.18$ & $\begin{array}{c}33.77 \pm 0.18 \\
(-11.15)\end{array}$ & $\begin{array}{c}32.76 \pm 0.48 \\
(-13.81)\end{array}$ \\
\hline E 24 & $94.66 \pm 1.14$ & $\begin{array}{c}92.66 \pm 1.67 \\
(-2.11)\end{array}$ & $\begin{array}{c}85.00 \pm 1.14 \\
(-10.20)\end{array}$ & $37.99 \pm 1.00$ & $\begin{array}{c}36.78 \pm 1.00 \\
(-3.19)\end{array}$ & $\begin{array}{c}36.38 \pm 0.34 \\
\quad(-4.24)\end{array}$ \\
\hline E 34 & $97.00 \pm 0.77$ & $\begin{array}{c}95.66 \pm 0.54 \\
(-1.38)\end{array}$ & $\begin{array}{c}91.33 \pm 0.77 \\
(-5.85)\end{array}$ & $39.69 \pm 0.48$ & $\begin{array}{c}37.28 \pm 0.87 \\
(-6.07)\end{array}$ & $\begin{array}{c}35.78 \pm 0.18 \\
(-9.85)\end{array}$ \\
\hline E 28 & $97.33 \pm 0.83$ & $\begin{array}{c}88.00 \pm 0.11 \\
(-9.59)\end{array}$ & $\begin{array}{c}77.33 \pm 1.20 \\
(-20.55)\end{array}$ & $40.04 \pm 2.11$ & $\begin{array}{c}37.47 \pm 1.28 \\
(-6.42)\end{array}$ & $\begin{array}{c}34.93 \pm 0.34 \\
(-12.76)\end{array}$ \\
\hline BAW1138 & $92.66 \pm 2.60$ & $\begin{array}{c}90.66 \pm 1.14 \\
(-2.16)\end{array}$ & $\begin{array}{c}83.33 \pm 2.60 \\
(-10.07)\end{array}$ & $40.45 \pm 0.48$ & $\begin{array}{c}36.89 \pm 0.76 \\
(-8.80)\end{array}$ & $\begin{array}{c}35.91 \pm 1.00 \\
(-11.22)\end{array}$ \\
\hline BAW1171 & $97.33 \pm 0.83$ & $\begin{array}{c}89.33 \pm 0.77 \\
(-8.22)\end{array}$ & $\begin{array}{c}87.33 \pm 0.54 \\
(-10.27)\end{array}$ & $43.62 \pm 1.25$ & $\begin{array}{c}40.27 \pm 1.00 \\
(-7.68)\end{array}$ & $\begin{array}{c}33.71 \pm 2.11 \\
(-22.72)\end{array}$ \\
\hline BAW1170 & $82.66 \pm 1.67$ & $\begin{array}{c}80.66 \pm 2.60 \\
(-2.42)\end{array}$ & $\begin{array}{c}70.00 \pm 0.11 \\
(-15.32)\end{array}$ & $40.81 \pm 0.72$ & $\begin{array}{c}38.72 \pm 0.34 \\
(-5.12)\end{array}$ & $\begin{array}{c}35.90 \pm 1.25 \\
(-12.03)\end{array}$ \\
\hline BAW1157 & $91.33 \pm 1.20$ & $\begin{array}{c}83.00 \pm 0.54 \\
(-9.12)\end{array}$ & $\begin{array}{c}80.66 \pm 1.14 \\
(-11.68)\end{array}$ & $40.04 \pm 0.72$ & $\begin{array}{c}36.55 \pm 1.00 \\
(-8.72)\end{array}$ & $\begin{array}{c}33.89 \pm 0.18 \\
(-15.36)\end{array}$ \\
\hline BAW1163 & $87.33 \pm 0.11$ & $\begin{array}{c}81.33 \pm 0.83 \\
(-6.87)\end{array}$ & $\begin{array}{c}73.66 \pm 1.20 \\
(-15.65)\end{array}$ & $34.50 \pm 1.00$ & $\begin{array}{c}29.00 \pm 2.11 \\
(-15.94)\end{array}$ & $\begin{array}{c}26.29 \pm 0.76 \\
(-23.80)\end{array}$ \\
\hline BAW1140 & $90.33 \pm 1.20$ & $\begin{array}{c}77.00 \pm 1.14 \\
(-14.76)\end{array}$ & $\begin{array}{c}63.33 \pm 2.60 \\
(-29.89)\end{array}$ & $40.34 \pm 0.76$ & $\begin{array}{c}33.23 \pm 1.25 \\
(-17.63)\end{array}$ & $\begin{array}{c}27.75 \pm 2.11 \\
(-31.21)\end{array}$ \\
\hline BAW1151 & $89.33 \pm 0.83$ & $\begin{array}{c}88.00 \pm 0.54 \\
(-1.49)\end{array}$ & $\begin{array}{c}75.00 \pm 1.67 \\
(-16.04)\end{array}$ & $38.06 \pm 0.72$ & $\begin{array}{c}37.02 \pm 1.00 \\
(-2.73)\end{array}$ & $\begin{array}{c}34.31 \pm 1.28 \\
(-9.85)\end{array}$ \\
\hline $\mathrm{CV}(\%)$ & & 2.08 & & & 1.65 & \\
\hline $\begin{array}{l}\text { Significance } \\
\text { level }\end{array}$ & & $* *$ & & & $* *$ & \\
\hline
\end{tabular}

Value within parenthesis indicates percent reduction from control, SE=standard error 
Table 3. Shoot length $(\mathrm{cm})$ and root length $(\mathrm{cm})$ of different wheat genotypes as influenced PEG induced water deficit stress

\begin{tabular}{|c|c|c|c|c|c|c|}
\hline \multirow[t]{2}{*}{ Genotypes } & \multicolumn{3}{|c|}{ Shoot length (mean \pm SE) } & \multicolumn{3}{|c|}{ Root length (mean \pm SE) } \\
\hline & $\begin{array}{c}\text { Control } \\
\text { (Tap water) }\end{array}$ & $\begin{array}{c}\text { Moderate } \\
\text { stress } \\
(-2 \text { bar }) \\
\end{array}$ & $\begin{array}{l}\text { Higher stress } \\
\text { (- 4bar) }\end{array}$ & $\begin{array}{c}\text { Control } \\
\text { (Tap water) }\end{array}$ & $\begin{array}{c}\text { Moderate } \\
\text { stress } \\
(-2 \mathrm{bar}) \\
\end{array}$ & $\begin{array}{c}\text { Higher } \\
\text { stress } \\
(-4 \mathrm{bar})\end{array}$ \\
\hline Satabdi & $23.18 \pm 0.37$ & $\begin{array}{l}17.41 \pm 0.15 \\
(-24.89)\end{array}$ & $\begin{array}{l}12.88 \pm 0.39 \\
(-44.43)\end{array}$ & $10.72 \pm 0.18$ & $\begin{array}{l}9.93 \pm 0.17 \\
(-7.37)\end{array}$ & $\begin{array}{l}8.86 \pm 0.31 \\
(-17.35)\end{array}$ \\
\hline Sourav & $23.78 \pm 0.43$ & $\begin{array}{l}15.43 \pm 0.29 \\
(-35.11)\end{array}$ & $\begin{array}{l}10.72 \pm 0.37 \\
(-54.92)\end{array}$ & $11.74 \pm 0.20$ & $\begin{array}{l}10.80 \pm 0.08 \\
(-8.01)\end{array}$ & $\begin{array}{l}8.44 \pm 0.28 \\
(-28.11)\end{array}$ \\
\hline $\begin{array}{l}\text { B ARI Gom } \\
25\end{array}$ & $20.73 \pm 0.15$ & $\begin{array}{l}17.74 \pm 0.15 \\
(-14.42)\end{array}$ & $\begin{array}{l}14.72 \pm 0.08 \\
(-28.99)\end{array}$ & $11.94 \pm 0.09$ & $\begin{array}{l}11.50 \pm 0.31 \\
(-3.69)\end{array}$ & $\begin{array}{l}10.46 \pm 0.18 \\
(-12.40)\end{array}$ \\
\hline $\begin{array}{l}\text { BARI Gom } \\
26\end{array}$ & $21.71 \pm 0.37$ & $\begin{array}{l}15.85 \pm 0.32 \\
(-26.99)\end{array}$ & $\begin{array}{l}10.72 \pm 0.13 \\
(-50.62)\end{array}$ & $12.44 \pm 0.17$ & $\begin{array}{l}10.72 \pm 0.20 \\
(-13.83)\end{array}$ & $\begin{array}{l}9.16 \pm 0.17 \\
(-26.37)\end{array}$ \\
\hline $\begin{array}{l}\text { BARI Gom } \\
27\end{array}$ & $23.63 \pm 0.32$ & $\begin{array}{l}18.54 \pm 0.28 \\
(-21.54)\end{array}$ & $\begin{array}{l}8.92 \pm 0.37 \\
(-62.25)\end{array}$ & $13.88 \pm 0.28$ & $\begin{array}{l}12.10 \pm 0.18 \\
(-12.82)\end{array}$ & $\begin{array}{l}10.8 \pm 0.40 \\
(-22.19)\end{array}$ \\
\hline $\begin{array}{l}\text { BARI Gom } \\
28\end{array}$ & $17.43 \pm 0.39$ & $\begin{array}{l}13.53 \pm 0.08 \\
(-22.37)\end{array}$ & $\begin{array}{l}8.25 \pm 0.43 \\
(-52.67)\end{array}$ & $12.10 \pm 0.18$ & $\begin{array}{l}11.15 \pm 0.31 \\
(-7.85)\end{array}$ & $\begin{array}{l}8.38 \pm 0.08 \\
(-30.74)\end{array}$ \\
\hline E 30 & $22.26 \pm 0.37$ & $\begin{array}{l}17.83 \pm 0.29 \\
(-19.90)\end{array}$ & $\begin{array}{l}10.41 \pm 0.15 \\
(-53.23)\end{array}$ & $10.90 \pm 0.20$ & $\begin{array}{l}10.00 \pm 0.40 \\
(-8.26)\end{array}$ & $\begin{array}{l}9.82 \pm 0.20 \\
(-9.91)\end{array}$ \\
\hline E 23 & $19.78 \pm 0.37$ & $\begin{array}{l}15.42 \pm 0.13 \\
(-22.04)\end{array}$ & $\begin{array}{l}10.33 \pm 0.39 \\
(-47.77)\end{array}$ & $11.96 \pm 0.17$ & $\begin{array}{l}11.58 \pm 0.13 \\
(-3.18)\end{array}$ & $\begin{array}{l}11.34 \pm 0.28 \\
(-5.18)\end{array}$ \\
\hline E 2 & $17.43 \pm 0.43$ & $\begin{array}{l}15.85 \pm 0.18 \\
(-9.06)\end{array}$ & $\begin{array}{l}9.86 \pm 0.29 \\
(-43.43)\end{array}$ & $12.95 \pm 0.18$ & $\begin{array}{l}12.47 \pm 0.31 \\
(-3.71)\end{array}$ & $\begin{array}{l}8.54 \pm 0.17 \\
(-34.05)\end{array}$ \\
\hline E 38 & $23.00 \pm 0.15$ & $\begin{array}{l}17.88 \pm 0.32 \\
(-22.26)\end{array}$ & $\begin{array}{l}11.97 \pm 0.37 \\
(-47.95)\end{array}$ & $12.51 \pm 0.18$ & $\begin{array}{l}12.28 \pm 0.40 \\
(-1.84)\end{array}$ & $\begin{array}{l}11.47 \pm 0.13 \\
(-8.31)\end{array}$ \\
\hline E 24 & $20.75 \pm 0.39$ & $\begin{array}{l}16.36 \pm 0.08 \\
(-21.15)\end{array}$ & $\begin{array}{l}11.82 \pm 0.43 \\
(-43.03)\end{array}$ & $11.09 \pm 0.40$ & $\begin{array}{l}10.26 \pm 0.18 \\
(-7.48)\end{array}$ & $\begin{array}{l}9.80 \pm 0.40 \\
(-11.63)\end{array}$ \\
\hline E 34 & $19.18 \pm 0.43$ & $\begin{array}{l}15.84 \pm 0.18 \\
(-17.41)\end{array}$ & $\begin{array}{l}11.98 \pm 0.15 \\
(-37.53)\end{array}$ & $12.73 \pm 0.28$ & $\begin{array}{l}12.31 \pm 0.18 \\
(-3.30)\end{array}$ & $\begin{array}{l}11.43 \pm 0.28 \\
(-10.21)\end{array}$ \\
\hline E 28 & $22.61 \pm 0.37$ & $\begin{array}{l}18.06 \pm 0.13 \\
(-20.12)\end{array}$ & $\begin{array}{l}15.54 \pm 0.43 \\
(-31.27)\end{array}$ & $11.61 \pm 0.31$ & $\begin{array}{l}10.22 \pm 0.13 \\
(-11.97)\end{array}$ & $\begin{array}{l}9.76 \pm 0.18 \\
(-15.93)\end{array}$ \\
\hline BAW1138 & $23.70 \pm 0.08$ & $\begin{array}{l}17.24 \pm 0.18 \\
(-27.25)\end{array}$ & $\begin{array}{l}10.46 \pm 0.15 \\
(-55.86)\end{array}$ & $12.12 \pm 0.17$ & $\begin{array}{l}11.12 \pm 0.20 \\
(-8.25)\end{array}$ & $\begin{array}{l}8.45 \pm 0.28 \\
(-30.28)\end{array}$ \\
\hline BAW1171 & $18.93 \pm 0.15$ & $\begin{array}{l}15.42 \pm 0.37 \\
(-18.54)\end{array}$ & $\begin{array}{l}9.88 \pm 0.39 \\
(-47.80)\end{array}$ & $13.34 \pm 0.40$ & $\begin{array}{l}12.50 \pm 0.08 \\
(-5.92)\end{array}$ & $\begin{array}{l}10.52 \pm 0.17 \\
(-21.14)\end{array}$ \\
\hline BAW1170 & $20.96 \pm 0.37$ & $\begin{array}{l}17.62 \pm 0.43 \\
(-15.94)\end{array}$ & $\begin{array}{l}15.47 \pm 0.29 \\
(-26.93)\end{array}$ & $12.48 \pm 0.28$ & $\begin{array}{l}11.34 \pm 0.09 \\
(-9.14)\end{array}$ & $\begin{array}{l}10.24 \pm 0.18 \\
(-17.95)\end{array}$ \\
\hline BAW1157 & $19.96 \pm 0.29$ & $\begin{array}{l}15.74 \pm 0.28 \\
(-21.14)\end{array}$ & $\begin{array}{l}9.88 \pm 0.32 \\
(-50.50)\end{array}$ & $13.67 \pm 0.08$ & $\begin{array}{l}12.27 \pm 0.31 \\
(-10.24)\end{array}$ & $\begin{array}{l}8.45 \pm 0.13 \\
(-38.19)\end{array}$ \\
\hline BAW1163 & $21.53 \pm 0.39$ & $\begin{array}{l}15.33 \pm 0.15 \\
(-28.79)\end{array}$ & $\begin{array}{l}10.65 \pm 0.37 \\
(-50.53)\end{array}$ & $9.94 \pm 0.20$ & $\begin{array}{l}8.98 \pm 0.18 \\
(-9.66)\end{array}$ & $\begin{array}{l}8.00 \pm 0.18 \\
(-19.52)\end{array}$ \\
\hline BAW1140 & $20.25 \pm 0.37$ & $\begin{array}{l}12.25 \pm 0.29 \\
(-39.51)\end{array}$ & $\begin{array}{l}7.32 \pm 0.37 \\
(-63.85)\end{array}$ & $11.08 \pm 0.17$ & $\begin{array}{l}9.00 \pm 0.28 \\
(-18.77)\end{array}$ & $\begin{array}{l}7.42 \pm 0.20 \\
(-33.03)\end{array}$ \\
\hline BAW1151 & $15.43 \pm 0.43$ & $\begin{array}{l}10.56 \pm 0.39 \\
(-31.56)\end{array}$ & $\begin{array}{l}10.37 \pm 0.15 \\
(-32.79)\end{array}$ & $10.86 \pm 0.18$ & $\begin{array}{l}9.10 \pm 0.13 \\
(-16.21)\end{array}$ & $\begin{array}{l}7.88 \pm 0.40 \\
(-27.44) \\
\end{array}$ \\
\hline $\mathrm{CV}(\%)$ & & 2.96 & & & 4.02 & \\
\hline $\begin{array}{l}\text { Significance } \\
\text { level }\end{array}$ & & $* *$ & & & $* *$ & \\
\hline
\end{tabular}

Value within parenthesis indicates percent reduction from control, $\mathrm{SE}=$ standard error 
Vigor index was found higher at control (ranging from 34.50 in BAW 1163 to 43.62 in BAW 1171 with a mean of 39.61 ) moderate at moderate stress (ranging from 29.00 in BAW 1163 to 40.86 in E 30 with a mean of 37.09) and lower at higher water deficit stress (with a range from 26.29 in BAW 1163 to 37.96 in BARI Gom 27 and a mean of 34.65). The results on vigor index showed that the speed of germination was reduced with the increment of water deficit stress but the degree of reduction was not similar for all wheat genotypes at moderate and higher water deficit stress compared to control. At moderate water deficit stress, wheat genotypes- BARI Gom 25, BARI Gom 27, E 30, E 2, E 24 and BAW 1151 showed less than $5 \%$ reduction and wheat genotypesSatabdi, Sourav, BARI Gom 26, BARI Gom 28, E 23, E 38, E 34, E 28, BAW 1138, BAW 1171, BAW 1170, BAW 1157, BAW 1163 and BAW 1140 showed more than $5 \%$ reduction in vigor index compared to control. At higher water deficit stress, wheat genotypes- Sourav, BARI Gom 25, BARI Gom 27, E 24, E 34 and BAW 1151 showed less than $10 \%$ reduction and wheat genotypes- Satabdi, BARI Gom 26, BARI Gom 28, E 30, E 23, E 2, E 38, E 28, BAW 1138, BAW 1171, BAW 1170, BAW 1157, BAW 1163 and BAW 1140 showed more than $10 \%$ reduction in vigor index compared to control.

As water is one of the primary requirements in seed germination (Shaban, 2013) the water stress developed by PEG reduced germination ability of seed greatly. Slower germination of wheat under water deficit stress was found due to lower surface contact of water with seed (Wuest et al., 1999) which restricts the water availability to the seeds (Soltani et al., 2002). Water deficit stress may also lead to degradation and inactivation of the essential hydrolytic and other group of enzymes required for germination (Pratab and Sharma, 2010). Differential degree of sensitivity in speed of germination to different water potentials was also found among the wheat genotypes. It may be due to genetic variability of wheat intolerance to water stress. Noorka and Khaliq (2007), Khayantnezhad et al. (2010) and Singh et al. (2008) also found differential sensitivity in germination velocity among different wheat genotypes in their studies.

\subsection{Early seedling growth \\ 3.2.1 Shoot length}

Shoot length of 10 days old seedling was significantly influenced by the interaction effect of water potential levels and wheat genotypes (Table 3). The shoot length was found to be higher at control (with a range from $15.43 \mathrm{~cm}$ in BAW 1151 to $23.78 \mathrm{~cm}$ in Sourav and a mean of $20.81 \mathrm{~cm}$ ), moderate at moderate stress (ranging from $10.56 \mathrm{~cm}$ in BAW 1151 to $18.54 \mathrm{~cm}$ in BARI Gom 27 with a mean of $16.00 \mathrm{~cm}$ ) and lower at higher water deficit stress (with a range from $7.32 \mathrm{~cm}$ in BAW 1140 to $15.54 \mathrm{~cm}$ in BAW 1138 and a mean of $11.11 \mathrm{~cm}$ ). The shoot length was found to be reduced with the increment of water deficit stress but the degree of reduction was not similar for all wheat genotypes. At moderate water deficit stress, wheat genotypesBARI Gom 25, E 30, E 2, E 34, BAW 1171and BAW 1170 showed less than $20 \%$ reduction and wheat genotypes-Satabdi, Sourav, E 30, E 2, E 38, E 24, BAW 1171, BAW 1157, BAW 1163 and BAW 1140 showed more than $20 \%$ reduction in shoot length compared to control. At higher water deficit stress, wheat genotypes- Satabdi, BARI Gom 25, E 23, E 34, E 2, E 38, E 24, E 28, BAW 1171, BAW 1170 and BAW 1151 showed less than $50 \%$ reduction and wheat genotypes- Sourav, BARI Gom 26, BARI Gom 27, BARI Gom 28, E 30, BAW 1138, BAW1157, BAW 1163 and BAW 1140 showed more than $50 \%$ reduction in shoot length compared to control.

\subsubsection{Root length}

Root length of seedling was significantly influenced by the interaction effect of water potential levels and wheat genotypes (Table 3). The root length was found to be higher at control (with a range from $9.94 \mathrm{~cm}$ in BAW 1163 to $13.88 \mathrm{~cm}$ in BARI Gom 27 and a mean of 12.00 $\mathrm{cm}$ ), moderate at moderate stress (ranging from $8.98 \mathrm{~cm}$ in BAW 1163 to $12.50 \mathrm{~cm}$ in BAW 1171 with a mean of $10.98 \mathrm{~cm}$ ) and lower at higher water deficit stress (with a range from $7.42 \mathrm{~cm}$ in BAW 1140 to $11.43 \mathrm{~cm}$ in E 34 and a 
mean of $9.46 \mathrm{~cm}$ ). The root length was found to be reduced with the increment of water deficit stress but the degree of reduction was not similar for all wheat genotypes. At moderate water deficit stress, wheat genotypes- Satabdi, Sourav, BARI Gom 25, BARI Gom 28, E 30, E 23, E 2, E 38, E 24, E 34, BAW 1138, BAW 1171, BAW 1170 and BAW 1163 showed less than $10 \%$ reduction and wheat genotypes- BARI Gom 26, BARI Gom 27, E 28, BAW 1157, BAW 1140 and BAW 1151 showed more than $10 \%$ reduction in root length compared to control. At higher water deficit stress, wheat genotypesSatabdi, BARI Gom 25, E 30, E 23, E 38, E 24, E 34, E 28, BAW 1170 and BAW 1163 showed less than $20 \%$ reduction and wheat genotypesSourav, BARI Gom 26, BARI Gom 27, BARI Gom 28, E 2, BAW 1138, BAW 1171, BAW 1157, BAW 1140 and BAW 1151showed more than $20 \%$ reduction in root length compared to control.

\subsubsection{Seedling dry weight}

Seedling dry weight was significantly influenced by the interaction effect of water potential levels and wheat genotypes (Table 4). The seedling dry weight was higher at control(from $83.66 \mathrm{mg}$ in BARI Gom 28 to $150.66 \mathrm{mg}$ in Satabdi), moderate at moderate stress (from $74.66 \mathrm{mg}$ in BARI Gom 28 to $116.33 \mathrm{mg}$ in Satabdi) and lower at higher water deficit stress (from 67.33 $\mathrm{mg}$ in E 24 to $100.00 \mathrm{mg}$ in BARI Gom 25). The seedling dry weight was reduced with the increment of water deficit stress but the degree of reduction was not similar for all wheat genotypes. At moderate water deficit stress, wheat genotypes- BARI Gom 25, BARI Gom 26, BARI Gom 27, BARI Gom 28, E 38, E 24, E 34, E 28, BAW 1171, BAW 1170 and BAW 1163 showed less than $20 \%$ reduction and wheat genotypes- Satabdi, Sourav, E 30, E 23, E 2, BAW 1138, BAW 1157, BAW 1140 and BAW 1151showed more than $20 \%$ reduction in seedling dry weight compared to control. At higher water deficit stress, wheat genotypesBARI Gom 25, BARI Gom 27, BARI Gom 28, E
30, E 34, E 28, BAW 1138, BAW 1171, BAW 1170 and BAW1157 showed less than 30\% reduction and wheat genotypes- Satabdi, Sourav, BARI Gom 26, E 23, E 2, E 38, E 24, BAW 1163, BAW 1140 and BAW 1151 showed more than $30 \%$ reduction in seedling dry weight compared to control.

Water deficit stress developed by PEG reduced the shoot length, root length and seedling dry weight of wheat genotypes in the present study. Similar results were found by Datta et al. (2011),Almaghrabi (2012), Khakwani et al. (2011), Raza et al. (2012) and Jajarmi (2009) in their studies. Because increasing the level of water stress not only inhibits the germination characteristics but the extension growth of the seedlings was also obstructed (Pratap and Sharma, 2010). Water stress also reduced the seed reserve utilization (Soltani et al., 2006 and Harb, 2013) solubilization of sugars (Harb, 2013) during germination which contributed to lower seedling dry weight of wheat. Different wheat genotypes showed differential degree in reduction in shoot length, root length and seedling dry weight in the present study. This may be due to differential genetic sensitivity of wheat genotypes to water deficit stress.

\subsubsection{Stress tolerance index based on seedling dry weight}

Stress tolerance index (STI) of twenty wheat genotypes based on seedling dry weight at moderate water deficit stress is presented in Figure 1. These STI values indicated a wide difference in water deficit stress tolerance among the wheat genotypes. Wheat genotypes- BARI Gom 25, E 34, E 28 and BAW 1170 showed more than 0.90 STI, the wheat genotypes-Sourav, E 23 and BAW 1140 provided less than 0.70 STI and the other wheat genotypes (Satabdi, BARI Gom 26, BARI Gom 27, BARI Gom 28, E 30, E 2, E 38, E 24, BAW 1138, BAW 1171, BAW 1157, BAW 1163 and BAW 1151) showed STI in between 0.70 to 0.90 . 
Table 4. Seedling dry weight (mg) of different wheat genotypes as influenced PEG induced water deficit stress

\begin{tabular}{|c|c|c|c|}
\hline \multirow[t]{2}{*}{ Genotypes } & \multicolumn{3}{|c|}{ Seedling dry weight (mean \pm SE) } \\
\hline & $\begin{array}{c}\text { Control } \\
\text { (Tap water) } \\
\end{array}$ & $\begin{array}{c}\text { Moderate stress } \\
\text { (- 2bar) }\end{array}$ & $\begin{array}{c}\text { Higher stress } \\
(-4 \mathrm{bar})\end{array}$ \\
\hline Satabdi & $150.66 \pm 0.61$ & $\begin{array}{c}116.33 \pm 0.52 \\
(-22.79)\end{array}$ & $\begin{array}{c}95.33 \pm 0.83 \\
(-36.73)\end{array}$ \\
\hline Sourav & $125.00 \pm 1.40$ & $\begin{array}{c}83.33 \pm 0.35 \\
(-33.34)\end{array}$ & $\begin{array}{c}83.33 \pm 0.76 \\
(-33.34)\end{array}$ \\
\hline BARI Gom 25 & $122.33 \pm 0.93$ & $\begin{array}{c}110.30 \pm 0.46 \\
(-9.83)\end{array}$ & $\begin{array}{c}100.00 \pm 0.11 \\
(-18.25)\end{array}$ \\
\hline BARI Gom 26 & $131.66 \pm 0.52$ & $\begin{array}{c}113.66 \pm 0.61 \\
(-13.67)\end{array}$ & $\begin{array}{c}89.66 \pm 1.40 \\
(-31.90)\end{array}$ \\
\hline BARI Gom 27 & $111.00 \pm 0.11$ & $\begin{array}{c}96.00 \pm 0.46 \\
(-13.51)\end{array}$ & $\begin{array}{c}83.66 \pm 0.93 \\
(-24.63)\end{array}$ \\
\hline BARI Gom 28 & $83.66 \pm 0.61$ & $\begin{array}{c}74.66 \pm 1.40 \\
(-10.76)\end{array}$ & $\begin{array}{c}68.33 \pm 0.76 \\
(-18.32)\end{array}$ \\
\hline E 30 & $115.33 \pm 0.83$ & $\begin{array}{c}86.33 \pm 0.58 \\
(-25.15)\end{array}$ & $\begin{array}{c}86.33 \pm 0.35 \\
(-25.15)\end{array}$ \\
\hline E 23 & $109.00 \pm 1.53$ & $\begin{array}{c}75.00 \pm 0.61 \\
(-31.19)\end{array}$ & $\begin{array}{c}68.00 \pm 0.52 \\
(-37.61)\end{array}$ \\
\hline E 2 & $105.00 \pm 0.93$ & $\begin{array}{c}77.66 \pm 1.40 \\
(-26.04)\end{array}$ & $\begin{array}{c}73.00 \pm 0.76 \\
(-30.48)\end{array}$ \\
\hline E 38 & $118.66 \pm 0.58$ & $\begin{array}{c}95.00 \pm 1.40 \\
(-19.94)\end{array}$ & $\begin{array}{c}78.66 \pm 0.61 \\
(-33.71)\end{array}$ \\
\hline E 24 & $102.33 \pm 1.53$ & $\begin{array}{c}84.33 \pm 0.93 \\
(-17.59)\end{array}$ & $\begin{array}{c}67.33 \pm 0.83 \\
(-34.20)\end{array}$ \\
\hline E 34 & $92.66 \pm 1.40$ & $\begin{array}{c}90.66 \pm 0.31 \\
(-2.16)\end{array}$ & $\begin{array}{c}68.66 \pm 0.33 \\
(-25.90)\end{array}$ \\
\hline E 28 & $97.00 \pm 0.76$ & $\begin{array}{c}88.33 \pm 0.52 \\
(-8.94)\end{array}$ & $\begin{array}{c}86.00 \pm 0.35 \\
(-11.34)\end{array}$ \\
\hline BAW1138 & $127.33 \pm 0.61$ & $\begin{array}{c}95.33 \pm 0.83 \\
(-25.13)\end{array}$ & $\begin{array}{c}96.00 \pm 1.53 \\
(-24.61)\end{array}$ \\
\hline BAW1171 & $116.33 \pm 0.33$ & $\begin{array}{c}96.66 \pm 0.93 \\
(-16.91)\end{array}$ & $\begin{array}{c}92.66 \pm 0.58 \\
(-20.35)\end{array}$ \\
\hline BAW1170 & $94.00 \pm 1.53$ & $\begin{array}{c}85.33 \pm 0.35 \\
(-9.22)\end{array}$ & $\begin{array}{c}84.66 \pm 1.40 \\
(-9.94)\end{array}$ \\
\hline BAW1157 & $116.00 \pm 0.52$ & $\begin{array}{c}89.33 \pm 1.40 \\
(-22.99)\end{array}$ & $\begin{array}{c}82.00 \pm 0.46 \\
(-29.31)\end{array}$ \\
\hline BAW1163 & $124.33 \pm 1.40$ & $\begin{array}{c}105.00 \pm 0.33 \\
(-15.55)\end{array}$ & $\begin{array}{c}78.66 \pm 0.11 \\
(-36.73)\end{array}$ \\
\hline BAW1140 & $136.00 \pm 0.46$ & $\begin{array}{c}83.00 \pm 0.58 \\
(-38.97)\end{array}$ & $\begin{array}{c}80.66 \pm 0.52 \\
(-40.69)\end{array}$ \\
\hline BAW1151 & $134.66 \pm 0.52$ & $\begin{array}{c}99.66 \pm 1.53 \\
(-25.99) \\
\end{array}$ & $\begin{array}{c}83.66 \pm 0.35 \\
(-37.87) \\
\end{array}$ \\
\hline $\mathrm{CV}(\%)$ & & 2.64 & \\
\hline $\begin{array}{l}\text { Significance } \\
\text { level }\end{array}$ & & $* *$ & \\
\hline
\end{tabular}

Value within parenthesis indicates percent reduction from control, $\mathrm{SE}=$ standard error 


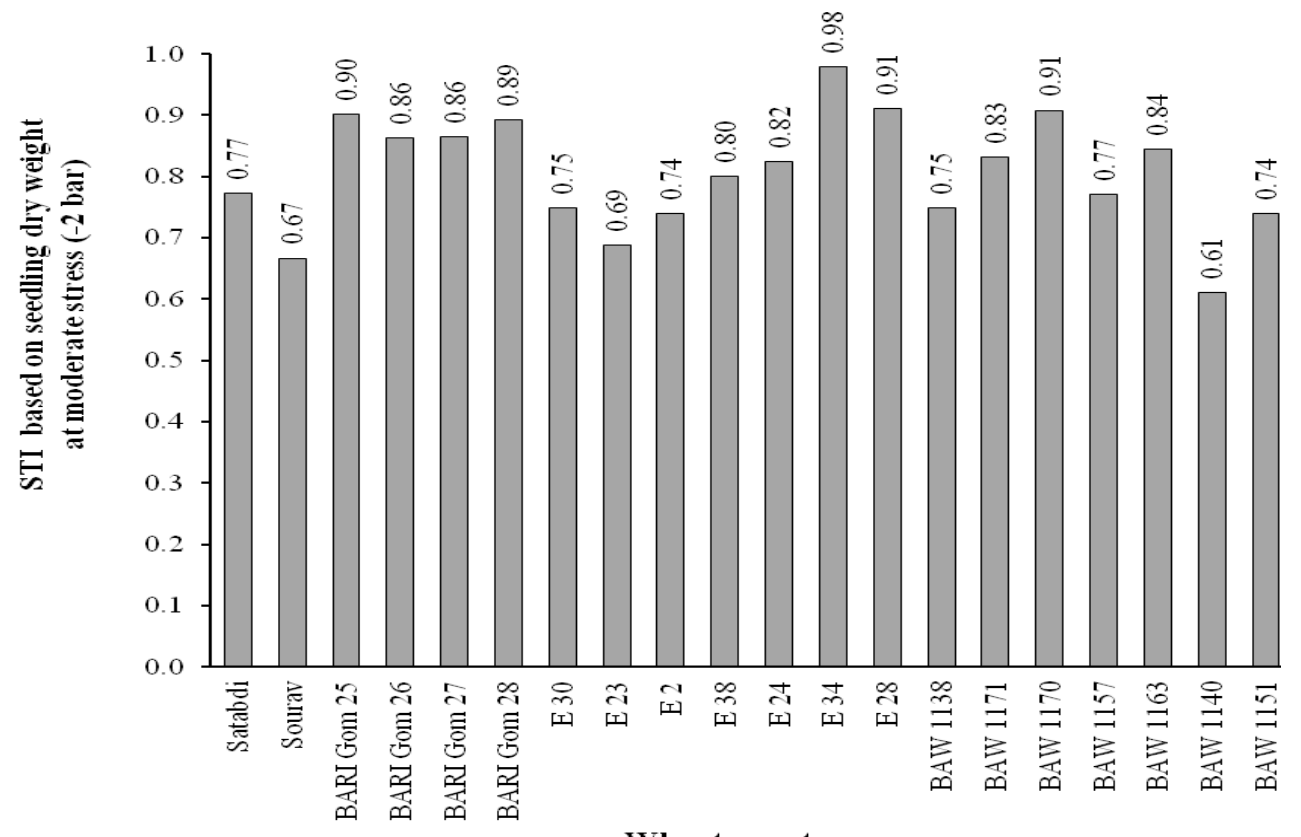

Figure 1. Stress tolerance index based on seedling dry weight of different wheat genotypes at moderate water deficit stress (- 2 bar)

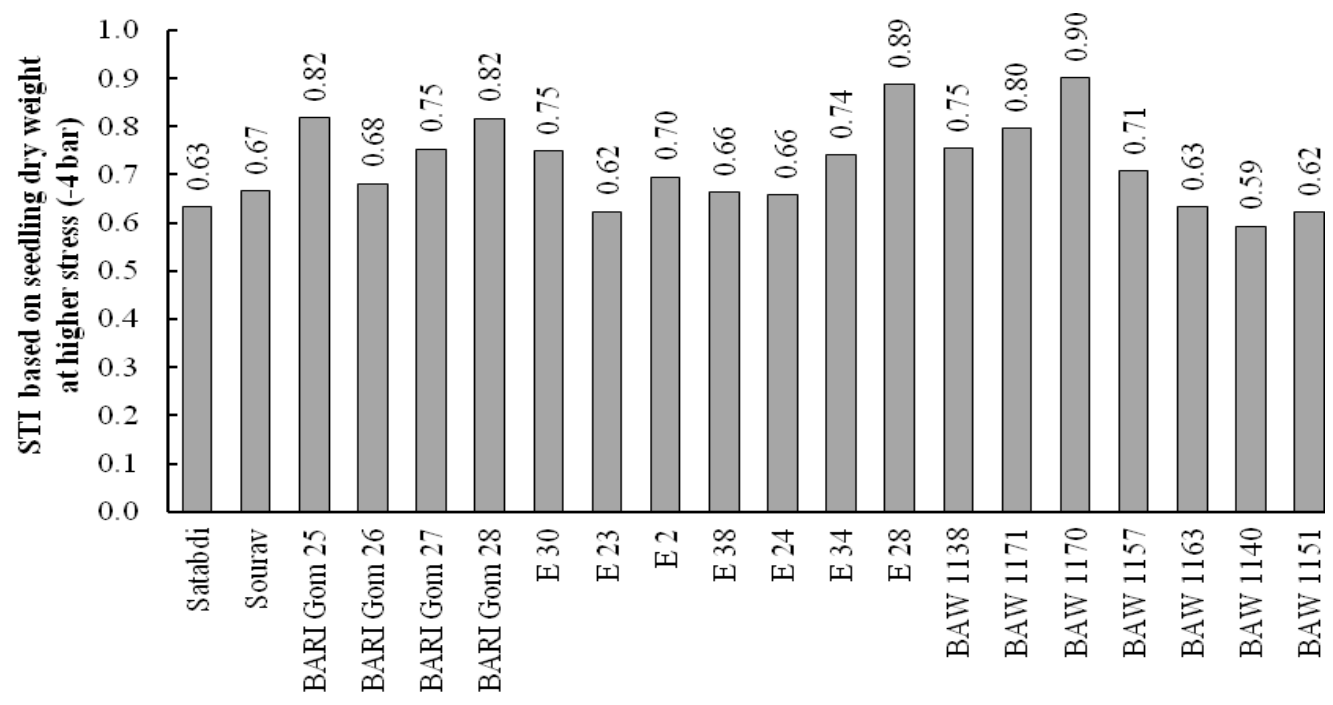

Wheat genotypes

Figure 2. Stress tolerance index based on seedling dry weight of different wheat genotypes at higher water deficit stress (- 4 bar) 
Figure 2. shows the stress tolerance index (STI) of twenty wheat genotypes based on seedling dry weight of at higher water deficit stress. These STI values also indicated a wide difference in water deficit stress tolerance among the wheat genotypes. Wheat genotypes- BARI Gom 25, BARI Gom 28, E 28 and BAW 1170 showed more than 0.80 STI, the wheat genotypesSatabdi, Sourav, BARI Gom 26, E 23, E 38, E 24, BAW 1163, BAW 1140 and BAW 1151 provided less than $0.70 \mathrm{STI}$ and the other wheat genotypes (BARI Gom 27, E 30, E 2, E 34, BAW 1138, BAW 1171 and BAW 1157) showed STI in between 0.70 to 0.80 .

\section{Conclusions}

Based on seedling dry weight, under both moderate and high water deficit stress BARI Gom 25, E 28 and BAW 1170 were found as tolerant and Sourav, E 23 and BAW 1140 were found as water deficit stress sensitive wheat genotypes during germination.

\section{References}

Almaghrabi, O. A. 2012. Impact of drought stress on germination and seedling growth parameters of some wheat cultivars. Life Science Journal, 9(1): 590-598.

Anonymous, 2014.Yearbook of agricultural statistics of Bangladesh, Bangladesh Bureau of Statistics, Ministry of planning and Government of the People's Republic of Bangladesh. 47 p.

Copeland, L. O. 1976. Principles of seed science and technology. Burgess Pub. Com., minneapolis, Minnesota, 164-165 pp.

Datta, J.K., Mondal, T., Banerjee, A. and Mondal, N. K. 2011.Assessment of drought tolerance of selected wheat cultivars under laboratory condition. Journal of Agricultural Technology, 7(2): 383-393.

Djibril, S. ,Mohamed, O.K., Diaga, D., Diegane, D., Abaya, B. F., Maurice, S. and
Alain,B.2005. Growth and development of date palm (Phoenix dactylifera L.) Seedlings under drought and salinity stresses. African Journal of Biotechnology, 4(9): 968-972.

Goudarzi, M. and Pakniyat, H. 2008.Evaluation of wheat cultivars under salinity stress based on some agronomic and physiological traits. Journal of Agriculture Society, 4: 35-38.

Harb, M. A. 2013. Reserve Mobilization, Total Sugars and Proteins in Germinating Seeds of Durum Wheat (Triticum durum Desf.) under Water Deficit after Short Period of Imbibition. Jordan Journal of Biological Sciences, 6(1): 67-72.

Hossain, A., Silva, J.A.T. 2013. Wheat production in Bangladesh: Its future in the light of global warming. AoB Plants, 5, 42 .

Jajarmi, V. 2009.Effect of water stress on germination indices in seven wheat cultivar. World Academy of Science, Engineering and Technology, 49: 105106.

Khakwani, A. A., Dennett, M. D. Munir, M. and Abid, M. 2011. Growth and yield response of wheat varieties to water stress at booting and anthesis stages of development. Pakistan Journal of Botany, 44(3): 879-886.

Khayantnezhad, M., Gholamin, R.Jamaati-eSomarin, S.andZabihi-e- Mahmooddabad, R. 2010.Study of water stress effect on wheat genotypes on germination indexes. Middle -East Journal of Scientific Reaserch, 6(6):657-660.

Krishnasamy, V. and Seshu, D. V.1990.Germination after accelerated aging and associated characters in rice varieties. Seed Science and Technology, 18: 353-359.

Lopez, C. G., Banowetz, G. M., Peterson, C. J. and Kronstad W. E. 2003.Dehydrin 
expression and drought tolerance in seven wheat cultivars. Crop Science. 43: 577582.

Michel, B. E. 1983. Evaluation of the Water Potentials of Solutions of Polyethylene Glycol 8000. Plant Physiology, 72:66-70.

Noorka, I. R. and Khaliq, I. 2007. An efficient technique for screening wheat (Triticum aestivum L.) germplasm for drought tolerance. Pakistan Journal of Botany, 39(5): 1539-1546.

Pratap, V. and Sharma, Y. K. 2010. Impact of osmotic stress on seed germination and seedling growth in black gram (Phaseolus mungo). Journal of Environmental Biology. 31(5): 721-726.

Rauf, M., Munir, M., Hassan, M., Ahmad, M. and Afzal, M. 2006.Performance of wheat genotypes under osmotic stress at germination and early seedling growth stage. African Journal of Biotechnology, 6: 971-975.

Raza,M. A. S., Saleem, M. F., Khan, I. H., Jamil, M.,Ijaz, M. and Khan, M. A. 2012. Evaluating the drought stress tolerance efficiency of wheat (Triticum aestivuml) Cultivars. Russian Journal of Agricultural and Socio-economic Sciences, 12(12): 4146.

Shaban, M. 2013. Effect of water and temperature on seed germination and emergence as a seed hydrothermal time model. International Journal of Advanced Biological and Biomedical Research, 1(12):1686-169.

Singh, G. P., Chaudhary, H. B., Rajbir, Y. And Tripathi, S. 2008. Genetic analysis of moisture stress tolerance in segregating populations of bread wheat (T. Aestivum L.). Indian Journal Agricultural Sciences, 78(10): 848-852.

Soltani, A., Galeshi, S., Zeinali, E.andLatifi, N. 2002. Germination, seed reserve utilization and seedling growth of chickpea as affected by salinity and seed size. Seed Science and Technology, 30: 51-60.

Soltani, A., Gholipoor, M. andZeinali, E. 2006. Seed reserve utilization and seedling growth of wheat as affected by drought and salinity. Environmental and Experimental Botany, 55:195-200.

Soltani, A., Galeshi, S., Zeinali, E. andLatifi,N. 2002. Germination, seed reserve utilization and seedling growth of chickpea as affected by salinity and seed size. Seed Science and Technology, 30: 51-60.

Wuest, S. B., Albrecht, S. L. and Skirvin, K. W. 1999. Vapor transport vs. seed- soil contact in wheat germination. Agronomy Journal, 91: 783-787. 